VOL. 48 (1993) [313-319]

\title{
THE INVARIANTS OF ORTHOGONAL GROUP ACTIONS
}

\author{
Li Chiang and Yu-Ching Hung
}

Let $F_{q}$ be the finite field of order $q$, an odd number, $Q$ a non-degenerate quadratic form on $F_{q}^{n}, O(n, Q)$ the orthogonal group defined by $Q$. Regard $O(n, Q)$ as a linear group of $F_{q}$-automorphisms acting linearly on the rational function field $F_{q}\left(x_{1}, \ldots, x_{n}\right)$. We shall prove that the invariant subfield $F_{q}\left(x_{1}, \ldots, x_{n}\right)^{O(n, Q)}$ is a purely transcendental extension over $F_{q}$ for $n=5$ by giving a set of generators for it.

\section{INTRODUCTION}

Let $F_{q}$ be the finite field of odd prime power order $q, Q\left(x_{1}, \ldots, x_{n}\right)$ a nondegenerate quadratic form on $V:=F_{q}^{n}$. The orthogonal group $O(n, Q)$ determined by $Q\left(x_{1}, \ldots, x_{n}\right)$ is defined as $\left\{\sigma \in G L\left(n, F_{q}\right): Q(\sigma \mathbf{v})=Q(\mathbf{v})\right.$ for all $\left.\mathbf{v} \in F_{q}^{n}\right\}$. Because a quadratic form is diagonalisable, $Q\left(x_{1}, \ldots, x_{n}\right)$ can be represented in one of the following forms:

$$
\begin{aligned}
& Q\left(x_{1}, \ldots, x_{n}\right)=x_{1}^{2}-x_{2}^{2}+x_{3}^{2}-\ldots-x_{n-1}^{2}-\varepsilon x_{n}^{2} \text { for odd } n \text {, } \\
& Q\left(x_{1}, \ldots, x_{n}\right)=x_{1}^{2}-x_{2}^{2}+x_{3}^{2}-\ldots+x_{n-1}^{2}-\epsilon x_{n}^{2} \text { for even } n \text {, }
\end{aligned}
$$

where $\varepsilon=1$ or $\varepsilon=\delta$, a nonsquare in $F_{q}$. Since $O(n, \delta Q)=O(n, Q), Q\left(x_{1}, \ldots, x_{n}\right)$ can be specified uniquely when $n$ is odd [4, Section 6.3,6.10]. The orthogonal group $O(n, Q)$, a subgroup of $G L\left(n, F_{q}\right)$, acts as a linear group of $F_{q}$-automorphisms on the polynomial ring $F_{q}\left[x_{1}, \ldots, x_{n}\right]$ and on the rational function field $F_{q}\left(x_{1}, \ldots, x_{n}\right)$ in a natural way.

For convenience, we introduce some notations defined as in $\left[3\right.$, p.217, 218]: $R_{n}:=$ $F_{q}\left[x_{1}, \ldots, x_{n}\right], K_{n}:=F_{q}\left(x_{1}, \ldots, x_{n}\right), K_{n}^{+}:=K_{n}^{O(n, Q)}, R_{n}^{+}:=R_{n}^{O(n, Q)}, Q_{n}(i):=$ $Q\left(x_{1}^{\left(q^{i}+1\right) / 2}, \ldots, x_{n}^{\left(q^{i}+1\right) / 2}\right)$ and $K_{n}^{*}:=F_{q}\left(Q_{n}(0), \ldots, Q_{n}(n-1)\right)$. It is noted in [2] that $Q_{n}(i)$ is invariant under $O(n, Q)$ for each $i \geqslant 0$. Thus, the invariant subfield $K_{n}^{+}$ contains $K_{n}^{*}$.

Received 28 October 1992

Partially supported by the National Science Council of the Republic of China under grant NSC 810208-M-003-04.

Copyright Clearance Centre, Inc. Serial-fee code: $0004-9729 / 93 \$ \$ 2.00+0.00$. 
In 1988, Chu [2] conjectured that $K_{n}^{+}$is exactly $K_{n}^{*}$ for all $n$, and that $K_{n}^{+}$is purely transcendental over $F_{q}$. Chu [2] has proved his conjecture for $n=2,3$. In 1989, Cohen [3] proved the case $n=4$. They also obtained the invariant subring $R_{n}^{+}$ for $n=2,3$. But they could not prove the conjecture for higher values of $n$. A full solution of this problem has been given in [1] by Carlisle and Kropholler, making use of Dickson's invariant theorem. In this note, we provide a proof for $n=5$ (Section 3) without using Dickson's invariant theorem. We also give a set of generators of the invariant subring $R_{4}^{+}$(Section 2, Theorem 3). Our main theorem is following:

THEOREM 1. For $n \leqslant 5, K_{n}^{+}=K_{n}^{*}$, which is purely transcendental over $F_{q}$.

2. The invariant subring of $O(4, Q)$ acting on $\boldsymbol{F}_{q}[\boldsymbol{x}, y, z, t]$

Through this section, $\delta$ denotes a fixed nonsquare in $F_{q}$. We let

$$
Q_{4}(x, y, z, t)=x^{2}+y^{2}-\delta z^{2}-t^{2} \quad \text { (a form in four variables). }
$$

For $Q_{2}(x, y)=x^{2}-\delta y^{2}, Q_{2}(2)$ and $Q_{2}(3)$ are in $R_{2}^{+}=F_{q}\left[Q_{2}(0), Q_{2}(1)\right]$ [2, Theorem 1]. Thus there exist two 2-variable polynomials $f(u, v)$ and $g(u, v)$ such that

$$
Q_{2}(2)=f\left(Q_{2}(0), Q_{2}(1)\right) \text { and } Q_{2}(3)=g\left(Q_{2}(0), Q_{2}(1)\right) \text {. }
$$

For $Q_{3}(x, y, z)=x^{2}+y^{2}-\delta z^{2}$ or $Q_{3}(x, y, z)=-x^{2}+y^{2}+\delta z^{2}$ we have the following theorem. The proof is similar to that of [3, Theorem 1.2].

ThEOREM 2. Let $Q_{3}(x, y, z)=x^{2}+y^{2}-\delta z^{2}$ or $Q_{3}(x, y, z)=-x^{2}+y^{2}+\delta z^{2}$. Then

$$
R_{3}^{+}=F_{q}\left[Q_{3}(0), Q_{3}(1), Q_{3}^{*}\right]
$$

where

$$
Q_{3}^{*}=\frac{Q_{3}(2)-Q_{3}(0)^{\left(q^{2}+1\right) / 2}}{Q_{3}(1)+Q_{3}(0)^{(q+1) / 2}} .
$$

In fact, by $[4$, Section 6.3$]$, the invariant ring $R_{3}^{+}$is the same as that in $[3$, Theorem $1.2]$.

Our main theorem in this section is following:

Theorem 3. Let $Q_{4}$ be defined by (1). Then $R_{4}^{+}=F_{q}\left[Q_{4}(0), Q_{4}(1), Q_{4}(2), Q_{4}^{*}\right]$ with

$$
Q_{4}^{*}=\frac{Q_{4}(3)-g\left(Q_{4}(0), Q_{4}(1)\right)}{Q_{4}(2)-f\left(Q_{4}(0), Q_{4}(1)\right)}
$$

where $f$ and $g$ are as in (2).

The following lemma is an important key to the proof of Theorem 3. 
Lemma 4. Let $a(u, v, w, s)$ be a polynomial in the polynomial ring $F_{q}[u, v, w, s]$. If $a\left(Q_{2}(0), Q_{2}(1), Q_{2}(2), Q_{2}(3)\right)=0$, then $a(u, v, w, s)$ is in the ideal generated by $A(u, v, w)$ and $B(u, v, s)$, where $A(u, v, w)=w-f(u, v) \in F_{q}[u, v, w, s]$, $B(u, v, s)=s-g(u, v) \in F_{q}[u, v, w, s]$ and $f, g$ are as in (2).

Proof: $A(u, v, w)$ and $B(u, v, s)$ are monic polynomials with respect to $w$ and s. Applying the division algorithm, we get

$$
a(u, v, w, s)=B(u, v, s) p(u, v, w, s)+A(u, v, w) q(u, v, w)+r(u, v)
$$

where $p(u, v, w, s), \quad q(u, v, w)$ and $r(u, v)$ are in $F_{q}[u, v, w, s]$. Because $a\left(Q_{2}(0), Q_{2}(1), Q_{2}(2), Q_{2}(3)\right)=0, \quad B\left(Q_{2}(0), Q_{2}(1), Q_{2}(3)\right)=0$ and $A\left(Q_{2}(0), Q_{2}(1), Q_{2}(2)\right)=0$, we have $r\left(Q_{2}(0), Q_{2}(1)\right)=0$. But $Q_{2}(0)$ and $Q_{2}(1)$ are algebraically independent over $F_{q}$, whence $r(u, v)=0$.

We now prove Theorem 3. Let $Q_{3}(x, y, z)=x^{2}+y^{2}-\delta z^{2} \in F_{q}[x, y, z]$. Because $Q_{3}(3) \in R_{3}^{+}$, by Theorem 2, there exists a three-variable polynomial $h_{1}$ such that $Q_{3}(3)=h_{1}\left(Q_{3}(0), Q_{3}(3), Q_{3}^{*}\right)$, where $Q_{3}^{*}$ is defined by (3), and the degree in the third variable of $h_{1}$ does not exceed $q+1[3,(4.5)]$. Therefore, there are another threevariable polynomial $h$ and some positive integer $d \leqslant q+1$ such that

$$
Q_{4}(3)\left[Q_{3}(1)+Q_{3}(0)^{(q+1) / 2}\right]^{d}+h\left(Q_{3}(0), Q_{3}(1), Q_{3}(2)\right)=0
$$

By [1] we can deduce that $d$ can be taken to be $q$, but we shall prove this ourselves. Substituting $Q_{3}(i)=x^{2}+y^{2}-\delta z^{2}=Q_{4}(i)+t^{2}$ into (3), we have

$$
\begin{gathered}
\left(Q_{4}(3)+t^{q^{3}+1}\right)\left[\left(Q_{4}(1)+t^{q+1}\right)+\left(Q_{4}(0)+t^{2}\right)^{(q+1) / 2}\right]^{d} \\
+h\left(Q_{4}(0)+t^{2}, Q_{4}(1)+t^{q+1}, Q_{4}(2)+t^{q^{3}+1}\right)=0 .
\end{gathered}
$$

Hence there is a polynomial

$$
\begin{gathered}
G(u, v, w, s, t)=\left(s+t^{q^{3}+1}\right)\left[\left(v+t^{q+1}\right)+\left(u+t^{2}\right)^{(q+1) / 2}\right]^{d} \\
+h\left(u+t^{2}, v+t^{q+1}, w+t^{q^{3}+1}\right)
\end{gathered}
$$

such that

$$
G\left(Q_{4}(0), Q_{4}(1), Q_{4}(2), Q_{4}(3), t\right)=0
$$

We write $G$ as

$$
G(u, v, w, s, t)=\sum_{k=0}^{M} a_{k}(u, v, w, s) t^{k}, \quad a_{M}(u, v, w, s) \neq 0 .
$$


Let $Q_{2}=y^{2}-\delta z^{2}$. There is no difference between $Q_{2}+t^{2}=y^{2}-\delta z^{2}+t^{2}$ and $Q_{4}+t^{2}=x^{2}+y^{2}-\delta z^{2}$ other than interchanging $t$ and $x$, thus

$$
G\left(Q_{2}(0), Q_{2}(1), Q_{2}(2), Q_{2}(3), t\right)=\sum_{k=0}^{M} a_{k}\left(Q_{2}(0), Q_{2}(1), Q_{2}(2), Q_{2}(3)\right) t^{k}=0
$$

Because $Q_{2}=y^{2}-\delta z^{2}$ is independent of $t$, then $a_{k}\left(Q_{2}(0), Q_{2}(1), Q_{2}(2), Q_{2}(3)\right)=0$ for all $k=0,1, \ldots, M$. By Lemma 4, all $a_{k}(u, v, w, s)$ must be in the ideal generated by $A(u, v, w)$ and $B(u, v, s)$. If the coefficient $a_{k}$ contains no $s$, then $A(u, v, w)$ is a factor of $a_{k}(u, v, w)$. Combining this with (5), we have

$$
\begin{gathered}
G(u, v, w, s, t)=[s-g(u, v)]\left[\left(v+t^{q+1}\right)+\left(u+t^{2}\right)^{(q+1) / 2}\right]^{d} \\
+[w-f(u, v)] h_{2}(u, v, w, t)
\end{gathered}
$$

for some polynomial $h_{2}(u, v, w, t) \in F_{q}[u, v, w, t]$. Now by (6),

$$
\begin{gathered}
{\left[Q_{4}(3)-g\left(Q_{4}(0), Q_{4}(1)\right)\right]\left[\left(Q_{4}(1)+t^{q+1}\right)+\left(Q_{4}(0)+t^{2}\right)^{(q+1) / 2}\right]^{d}} \\
=-\left[Q_{4}(2)-f\left(Q_{4}(0), Q_{4}(1)\right)\right] h_{2}\left(Q_{4}(0), Q_{4}(1), Q_{4}(2), t\right)
\end{gathered}
$$

Then

$$
\begin{aligned}
Q_{4}(2)- & f\left(Q_{4}(0), Q_{4}(1)\right) \mid\left[Q_{4}(3)-g\left(Q_{4}(0), Q_{4}(1)\right)\right]\left[\left(Q_{4}(1)+t^{q+1}\right)\right. \\
& \left.+\left(Q_{4}(0)+t^{2}\right)^{(q+1) / 2}\right]^{d} .
\end{aligned}
$$

(Notation: $a \mid b$ means $a$ is a factor of $b$.) Applying the same method and replacing $Q=x^{2}+y^{2}-\delta z^{2}$ with $Q=-x^{2}+t^{2}+\delta z^{2}$, we get that

$$
\begin{aligned}
Q_{4}(2)- & f\left(Q_{4}(0), Q_{4}(1)\right) \mid\left[Q_{4}(3)-g\left(Q_{4}(0), Q_{4}(1)\right)\right]\left[\left(-Q_{4}(1)+y^{q+1}\right)\right. \\
& \left.+\left(-Q_{4}(0)+y^{2}\right)^{(q+1) / 2}\right]^{d} .
\end{aligned}
$$

By symmetry, we also get

$$
\begin{aligned}
Q_{4}(2)- & f\left(Q_{4}(0), Q_{4}(1)\right) \mid\left[Q_{4}(3)-g\left(Q_{4}(0), Q_{4}(1)\right)\right]\left[\left(-Q_{4}(1)+x^{q+1}\right)\right. \\
+ & \left.\left(-Q_{4}(0)+x^{2}\right)^{(q+1) / 2}\right]^{d} .
\end{aligned}
$$

Since there is no common non-unit factor of $\left[\left(-Q_{4}(1)+y^{q+1}\right)+\left(-Q_{4}(0)+y^{2}\right)^{(q+1) / 2}\right]^{d}$, $\left[\left(-Q_{4}(1)+x^{q+1}\right)+\left(-Q_{4}(0)+x^{2}\right)^{(q+1) / 2}\right]^{d}$ and $\left[\left(Q_{4}(1)+t^{q+1}\right)+\left(Q_{4}(0)+t^{2}\right)^{(q+1) / 2}\right]^{d}$, we have

$$
Q_{4}(2)-f\left(Q_{4}(0), Q_{4}(1)\right) \mid\left[Q_{4}(3)-g\left(Q_{4}(0), Q_{4}(1)\right)\right]
$$

Hence, we conclude that 


$$
Q_{4}^{*}=\frac{Q_{4}(3)-g\left(Q_{4}(0), Q_{4}(1)\right)}{Q_{4}(2)-f\left(Q_{4}(0), Q_{4}(1)\right)} \in F_{q}[x, y, z, t]
$$

and also that

$$
Q_{4}(2)-f\left(Q_{4}(0), Q_{4}(1)\right) \mid a_{k}\left(Q_{4}(0), Q_{4}(1), Q_{4}(2), Q_{4}(3)\right)
$$

for all $k=0, \ldots, M$. Moreover, if we set $t=0$ or $y=0$ in (8) we get

$$
Q_{3}(2)-f\left(Q_{3}(0), Q_{3}(1)\right) \mid Q_{3}(3)-g\left(Q_{3}(0), Q_{3}(1)\right)
$$

for the quadratic form $Q_{3}=x^{2}+y^{2}-\delta z^{2}$ or $Q_{3}=-x^{2}+t^{2}+\delta z^{2}$. By [3, Lemma 2.4], we have

$$
w-f(u, v) \equiv\left(w-u^{\left(q^{2}+1\right) / 2}\right) \quad\left(\bmod v+u^{(q+1) / 2}\right) .
$$

But, by Theorem 2, we have

$$
Q_{3}(1)+Q_{3}(0)^{(q+1) / 2} \mid Q_{3}(2)-Q_{3}(0)^{\left(q^{2}+1\right) / 2} .
$$

Then

$$
Q_{3}(1)+Q_{3}(0)^{(q+1) / 2} \mid Q_{3}(2)-f\left(Q_{3}(0), Q_{3}(1)\right)
$$

and therefore $Q_{3}(1)+Q_{3}(0)^{(q+1) / 2}$ is a factor of $Q_{3}(3)-g\left(Q_{3}(0), Q_{3}(1)\right)$. So,

$$
\frac{Q_{3}(3)-g\left(Q_{3}(0), Q_{3}(1)\right)}{Q_{3}(1)+Q_{3}(0)^{(q+1) / 2}} \in R_{3}^{+}=F_{q}\left[Q_{3}(0), Q_{3}(1), Q_{3}^{*}\right] .
$$

Then

$$
Q_{3}(3)-g\left(Q_{3}(0), Q_{3}(1)\right)=\left(Q_{3}(1)+Q_{3}(0)^{(q+1) / 2}\right) h_{3}\left(Q_{3}(0), Q_{3}(1), Q_{3}^{*}\right)
$$

where the polynomial $h_{3}$ is in $F_{q}\left[Q_{3}(0), Q_{3}(1), Q_{3}^{*}\right]$ and the degree in $Q_{3}^{*}$ of $h_{3}$ does not exceed $q+1$. This can be proved by counting the homogeneous degrees. Comparing (4) and (9), we conclude that $d \leqslant q$ if we minimise $d$. By [3, Theorem 1.1] and [4, Theorem $6.17], t$ has $\left[K_{3}^{+}(t): K_{4}^{+}\right]=\left[K_{4}: K_{4}^{+}\right] /\left[K_{4}: K_{3}^{+}(t)\right]=\left|O\left(4, Q_{4}\right)\right| /\left|O\left(3, Q_{3}\right)\right|=$ $q^{3}+q$ conjugates in $K_{4}$ over $K_{4}^{+}$. Thus the highest power of $t$ in the polynomial $G(u, v, w, s, t)$ is not less than $q^{3}+q$.

Now if we take $d=q$, the leading coefficient $a_{M}$ of $t$ in (7) is a homogeneous polynomial for variables $x, y, z, t$ with degree not greater than $q^{3}+1+q(q+1)-$ $\left(q^{3}+q\right)=q^{2}+1$.

On the other hand, all coefficients $a_{k}$ in (7) are divisible by $Q_{4}(2)-f\left(Q_{4}(0), Q_{4}(1)\right)$, a homogeneous polynomial with degree $q^{2}+1$, and hence $a_{M}(u, v, w, s)=$ 
$\gamma(w-f(u, v))$ for some unit $\gamma \in F_{q}$. Dividing $G(u, v, w, s, t)$ by $\gamma(w-f(u, v))$, we get a monic polynomial of $t$ in $F_{q}[u, v, w,(s-g(u, v)) /(w-f(u, v)), t]$. This implies that $t$ is integral over

$$
R:=F_{\mathrm{q}}\left[Q_{4}(0), Q_{4}(1), Q_{4}(2), \frac{Q_{4}(3)-g\left(Q_{4}(0), Q_{4}(1)\right)}{Q_{4}(2)-f\left(Q_{4}(0), Q_{4}(1)\right)}\right] .
$$

By the same process $x$ and $y$ are integral over $R$ and, hence, so is $z$.

Now $R_{4}=F_{q}[x, y, z, t]$ is integral over $R$ and $R \subset R_{4}^{+} \subset R_{4}$, so $R_{4}^{+}$is also integral over $R$. Since $R$, a unique factorisation domain, is integrally closed in its field of quotients $Q(R)=K_{4}^{*}=K_{4}^{+}$and $R \subset R_{4}^{+} \subset Q(R), R$ is also integrally closed in $R_{4}^{+}$. Thus $R_{4}^{+}=R$. This completes the proof of Theorem 3 .

\section{THE PROOF OF THEOREM 1}

We now prove Theorem 1. Consider $Q_{4}(4) \in R_{4}^{+}$, where $Q_{4}(x, y, z, t)$ is defined by (1). By Theorem 3 we have a four-variable polynomial $h_{4}$ such that

$$
Q_{4}(4)-h_{4}\left(Q_{4}(0), Q_{4}(1), Q_{4}(2), Q_{4}^{*}\right)=0 .
$$

$Q_{4}^{*}$ is a homogeneous polynomial in $F_{q}[x, y, z, t]$ of degree $q^{3}+1-\left(q^{2}+1\right)=q^{3}-q^{2}$ and $Q_{4}(4)$ has degree $q^{4}+1$. Thus, the degree in $Q_{4}^{*}$ does not exceed

$$
\frac{q^{4}+1}{q^{3}-q^{2}}<q+2
$$

for $q \geqslant 3$. Hence, multiplying $(10)$ by $\left[Q_{4}(2)-f\left(Q_{4}(0), Q_{4}(1)\right)\right]^{q+1}$, we get

$$
Q_{4}(4)\left[Q_{4}(2)-f\left(Q_{4}(0), Q_{4}(1)\right)\right]^{q+1}-h_{5}\left(Q_{4}(0), Q_{4}(1), Q_{4}(2), Q_{4}(3)\right)=0 .
$$

By [3, Section 6.3], we may assume that our five-variable quadratic form is

$$
Q_{5}=x^{2}+y^{2}-\delta z^{2}-t^{2}-\theta^{2},
$$

where $\delta$ is a non-square in $F_{q}$. Substituting $Q_{4}(i)=Q_{5}(i)+\theta^{q^{i}+1}, i=0,1,2,3,4$ into (11), we obtain

$$
\begin{aligned}
& \left(Q_{5}(4)+\theta^{q^{4}+1}\right)\left[\left(Q_{5}(2)+\theta^{q^{2}+1}\right)-f\left(Q_{5}(0)+\theta^{2}, Q_{5}(1)+\theta^{q+1}\right)\right]^{q+1} \\
& \quad-h_{5}\left(Q_{5}(0)+\theta^{2}, Q_{5}(1)+\theta^{q+1}, Q_{5}(2)+\theta^{q^{2}+1}, Q_{5}(3)+\theta^{q^{3}+1}\right)=0 .
\end{aligned}
$$

Let $L:=F_{q}\left(Q_{4}(0), Q_{4}(1), Q_{4}(2), Q_{4}(3), \theta\right)=K_{5}^{*}(\theta)$. Then it is clear that

$$
F_{q}(x, y, z, t, \theta)=K_{5} \supset L=K_{4}^{+}(\theta) \supset K_{5}^{*}=F_{q}\left(Q_{5}(0), Q_{5}(1), Q_{5}(2), Q_{5}(3), Q_{5}(4)\right) \text {. }
$$


Let $m:=\left[L: K_{5}^{*}\right]$. Then, by (12), $m \leqslant q^{4}+1+(q+1)\left(q^{2}+1\right)<2 q^{2}\left(q^{2}-1\right)$ for $q \geqslant 3$.

Because the Galois group of $K_{5}$ over $L$ is isomorphic to $O\left(4, Q_{4}\right)$ and the Galois group of $K_{5}$ over $K_{5}^{+}$is isomorphic to $O\left(5, Q_{5}\right)$, we have $\left[K_{5}: L\right]=\left|O\left(4, Q_{4}\right)\right|$ and $\left[K_{5}: K_{5}^{+}\right]=\left|O\left(5, Q_{5}\right)\right|$. Hence

$$
\left[K_{5}: K_{5}^{*}\right]=m\left|O\left(4, Q_{4}\right)\right|<2 q^{2}\left(q^{2}-1\right)\left|O\left(4, Q_{4}\right)\right|=4 q^{4}\left(q^{2}-1\right)^{2}\left(q^{2}+1\right) .
$$

Since $K_{5} \supset K_{5}^{+} \supset K_{5}^{*},\left[K_{5}: K_{5}^{+}\right]=\left|O\left(5, Q_{5}\right)\right|=2 q^{4}\left(q^{2}-1\right)^{2}\left(q^{2}+1\right)$ is a divisor of $\left[K_{5}: K_{5}^{*}\right]$. Thus $\left[K_{5}: K_{5}^{+}\right]=2 q^{4}\left(q^{2}-1\right)^{2}\left(q^{2}+1\right)=\left[K_{5}: K_{5}^{*}\right]$. So we get $K_{5}^{+}=K_{5}^{*}$. This completes the proof of Theorem 1 .

\section{REFERENCES}

[1] D. Carlisle and P.H. Kropholler, 'Rational invariants of certain orthogonal and unitary groups', (preprint).

[2] H. Chu, 'Orthogonal group action on rational functions fields', Bull. Inst. Math. Acad. Sinica 16 (1988), 115-122.

[3] S.D. Cohen, 'Rational function invariant under an orthogonal group', Bull. London Math. Soc. 22 (1990), 217-221.

[4] N. Jacobson, Basic algebra $I$, 1st ed. (Freeman and Company, New York, San Francisco, 1974).

Department of Mathematics

National Taiwan Normal University

Taipei

Taiwan

Republic of China 